\title{
TWO APPLICATIONS OF WAVELET ANALYSIS FOR PROJECT LEVEL PAVEMENT MANAGEMENT
}

\author{
R. HASSAN \\ Faculty of Science, Engineering and Technology, Centre for Sustainable Infrastructure, \\ Swinburne University of Technology, Australia.
}

\begin{abstract}
Wavelet analysis is a signal processing technique that can be used to decompose longitudinal road surface profile signal into a number of wavebands. The outputs of the analysis include the signals (elevation vs. distance) and energies (a measure of elevation variation, i.e. surface roughness) in the different wavebands. The application of wavelet analysis in road pavement management at project level is described herein through two case studies. The first involves using wavelet analysis outputs in identifying and locating excitation sources of dynamic wheel loads (DWL) along a road section. The second case study involves using these outputs in assessing the effectiveness of rehabilitation treatment in reducing surface roughness in the different wavebands along the length of a road section. The outcomes of this research study indicate that the proposed applications are effective in addressing the intended purposes. Study findings also indicate that using HATI to highlight sections subject to high DWL at network level is promising. However, further testing is required to confirm its suitability at different speeds and operating environments.

These assessment approaches help asset managers to identify and locate surface characteristics that increase pavement damage, propose suitable treatments and assess the quality of these treatments. In addition to achieving value for money, adopting such approach would ensure their assets' sustainability, mobility and comfort of all road users, in particular truck drivers. Long wavelength roughness with high contributions to DWL also has a detrimental effect on heavy vehicle ride.

Keywords: Dynamic wheel loads, granular overlay, heavy articulated truck index, profile data, road pavements, roughness, simulation, wavelet analysis.
\end{abstract}

\section{INTRODUCTION}

The objective of the study reported herein is to demonstrate the application of wavelet analysis through two case studies related to project level pavement asset management. The first case study demonstrates the use of wavelet analysis for identifying and locating sources of excitation for dynamic wheel loads (DWL). By identifying DWL excitation sources, asset managers can determine appropriate remedial measures to eliminate the additional pavement damage caused by DWL and increase its service life. The second case study demonstrates the use of DWT analysis outputs in assessing effectiveness of rehabilitation treatment. Commonly, asset managers use condition data to assess the effectiveness of applied treatments. However, such approach does not always prove sufficient in assessing the quality of works applied as demonstrated in this limited study.

For comparison purposes, two profile-based roughness indices currently used by State Road Agency in Victoria/Australia are considered. They include the International Roughness index (IRI) and Heavy Articulated Truck Index (HATI). Both indices are used at network management level to trigger investigation into pavement rehabilitation to improve ride quality. The intervention levels for these measures are $4.5 \mathrm{~m} / \mathrm{km}$ for IRI and $1.7 \mathrm{~km} / \mathrm{hr}$ for HATI. HATI is used for identifying pavement sections providing poor ride quality for heavy vehicle drivers. It is determined the same way as the IRI but the profile data for a section are processed through a quarter truck model (at $100 \mathrm{~km} / \mathrm{hr}$ ) instead of the IRI quarter car model, at $80 \mathrm{~km} / \mathrm{hr}$. The $100 \mathrm{~km} / \mathrm{hr}$ represents speed limit for truck on State highways in Victoria. Details of HATI, its development and validation can be found in Hassan and McManus [1]. 


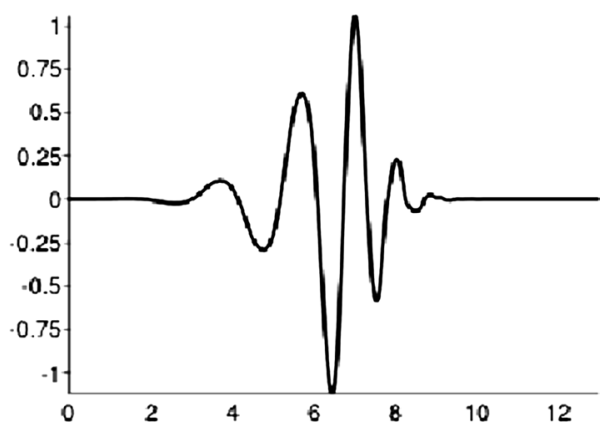

Figure 1: DB7 [6].

\subsection{Wavelet analysis}

Discrete wavelet transform (DWT) is a signal processing tool. It decomposes the longitudinal road surface profile signal into a number of consecutive wavebands and has been used in a number of studies to evaluate road roughness characteristics [2-6]. The first step in performing DWO is to select a suitable mother wavelet for the decomposition. Daubechies (DB) wavelets have been used by many researchers in decomposing road surface profile signal. DB wavelets range from 1 to 20 wavelets with high order ones being smoother than low order ones [7]. In this study, a Daubechies wavelet of the order of seven (DB7), see Fig. 1, was used as it was found to be suitable for the purpose of this study in terms of accuracy in both frequency and distance domains.

The minimum wavelength detectable from wavelet analysis depends on the sampling interval of profile signal. The level of decomposition achievable depends on the number of the data points. The sampling interval for profile data, used herein, is $0.05 \mathrm{~m}$, so the minimum wavelength detectable is $0.1 \mathrm{~m}$. The maximum level of decomposition can be expressed by $2 \mathrm{n}$ and $2 \mathrm{n} \leq \mathrm{N}$, where (n) is the level of decomposition and $(\mathrm{N})$ is the number of data points in the record. For a segment length of $100 \mathrm{~m}$, the number of data points obtained from a sampling interval of $0.05 \mathrm{~m}$ is 2,000. Therefore, a maximum of 10 levels $(210=1,024$ points $)$ of decomposition (D1-D10) can be obtained for this length. The ranges of wavelengths in each level or waveband (D) are: D1 $(0.1-0.2 \mathrm{~m}), \mathrm{D} 2(0.2-0.4 \mathrm{~m})$, D3 (0.4-0.8 m), D4 (0.8-1.6 m), D5 (1.6-3.2 m), D6 (3.2-6.4m), D7 (6.4-12.8m), D8 (12.8-25.6m), D9 (25.6-51.2m) and D10 (51.2-102 m).

DWT analysis outputs include the signals (elevation vs. distance) in the different wavebands and associated energies. This makes its use in pavement management more attractive than other signal processing tools such as Power Spectral Density analysis, where surface defects can be located along the length of a section. The energy measure in each waveband represents the extent of variation of pavement profile elevation in that waveband [6]. These outputs are generated separately for the profile data in the inner and outer wheel paths. A typical plot of wavelet signals in the wavebands (D2-D8) for the outer wheel path of a 90-m section is presented in Fig. 2.

\section{CASE STUDY 1: IDENTIFY AND LOCATE SOURCES OF EXCITATION FOR DYNAMIC WHEEL LOADS}

Many studies [8-12] have shown that the interaction between road surface roughness and heavy vehicle body results in DWL, which increase pavement damage. The magnitude of DWL is dependent on road surface profile characteristics, travel speed and heavy vehicle properties, particularly the types of suspension systems. 

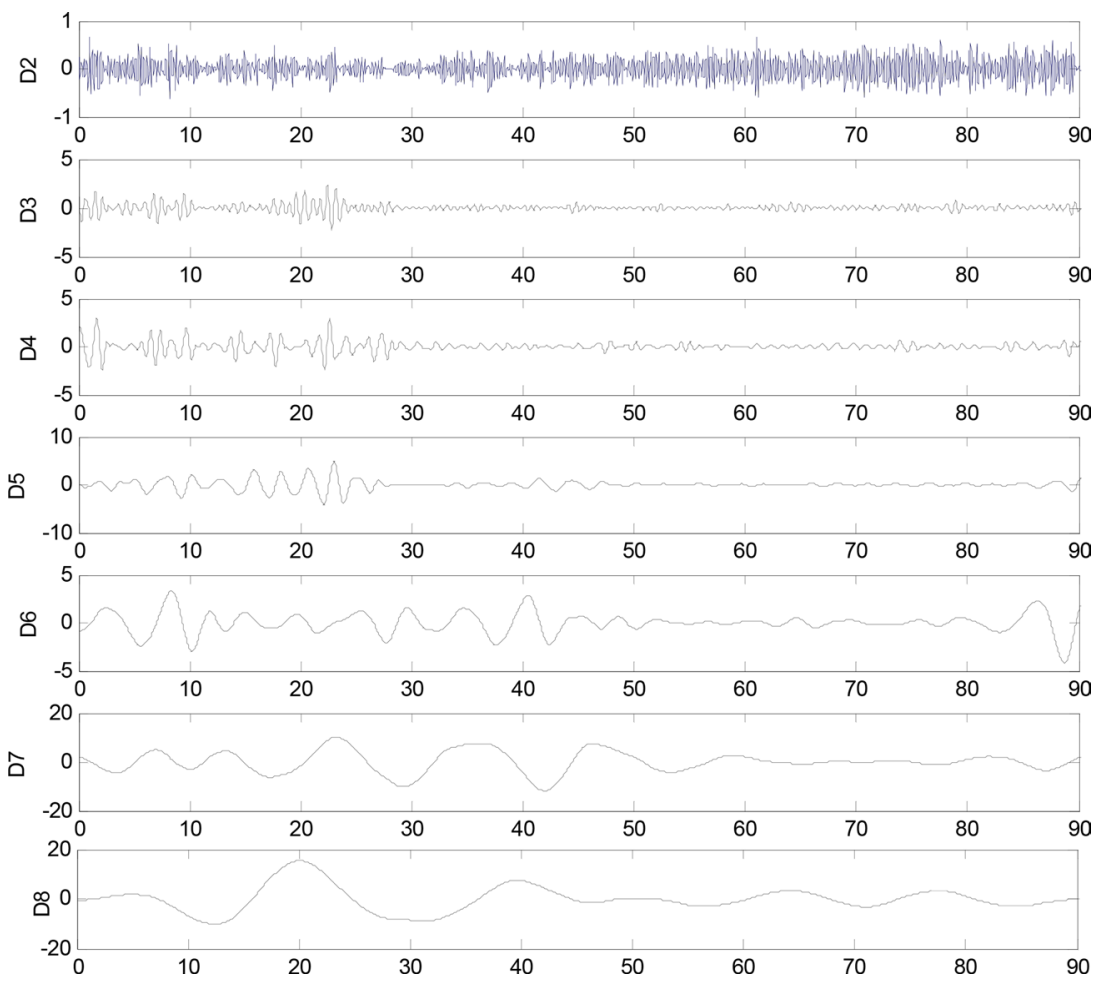

Figure 2: Decomposed signal of a section for wavebands D2-D8. The horizontal axis is distance $(\mathrm{m})$ and the vertical axis is elevation $(\mathrm{m})$.

Considering the different available suspension and vehicle types, the excitation frequencies of DWL generally fall into two ranges, low and high $[9,10]$.

- The low frequency range is $1.5-4 \mathrm{~Hz}$, which corresponds to rigid body bounce and pitch modes.

- The high frequency range is $8-15 \mathrm{~Hz}$ and corresponds to rigid body hop mode, axle roll and load sharing suspension pitch modes.

At highway speeds (80-100 km/hr) along major freight routes in Victoria/Australia, longitudinal road profile wavelengths that cause excitations of DWL at low and high frequencies fall in the range of $(5.6-18.5 \mathrm{~m})$ and $(1.5-3.5 \mathrm{~m})$, respectively. However, many studies [9-12] have shown that the lower frequency vibration modes are the major contributors to DWL for all suspension types.

In this case study, the profile data of 10 sections are used for DWT analysis and as input for simulating DWL applied by a typical truck. The sections are from three major freight routes in rural and all have granular flexible pavements with sprayed/chip seals. Their lengths range between $100 \mathrm{~m}$ and $600 \mathrm{~m}$, and their IRI and HATI values range between 1.7 and $5.18 \mathrm{~m} / \mathrm{km}$, and 0.58 and $3.09 \mathrm{~m} / \mathrm{km}$, respectively. Considering that DWL simulation packages are not normally available to pavement asset managers, the use of HATI and IRI as indicators of sections subject to high DWL at network level is also tested. 


\subsection{DWL and HATI}

The simulation is conducted using a full vehicle model of a five-axle tractor-semi-trailer combination unit. It had tandem drive and trailer axles with dual tyres (see Fig. 3) and both are fitted with air bag suspensions. The vehicle is fully laden with a gross mass of 39 tonnes. It complies with Australian heavy vehicle mass and dimensions regulations and constitutes a reasonable proportion of the truck fleet in Victoria. The simulation speed is $100 \mathrm{~km} / \mathrm{hr}$, which is the speed limit for trucks on rural highways in Victoria. The simulation is performed using a customised version of TruckSim.

The simulation outputs include the distribution of dynamic wheel forces (in Newtons) associated with all five axles in the outer and inner wheel paths, sampled at 0.005 -second intervals. These DWL signals are then used to identify excitation frequencies with high contributions by plotting their spectrum using Matlab. The DWL spectra of all test sections show that the frequency band where the peak forces could be observed is $0.5-3 \mathrm{~Hz}$. Low-frequency modes are excited by the long wavelengths of the roughness spectrum. However, the contribution from the roll motion that occurs at frequencies below $1 \mathrm{~Hz}$ is related to the effects of road crossfall variation and/or differences in elevation and direction of movement between the outer and inner wheel paths. The latter is true for all 10 test sections.

The dynamic wheel forces generated along each section by the tyres of all five axles, in both wheel paths, are also used to estimate the Dynamic Aggregate Force Coefficient (DAFC). The DAFC is a measure that characterises the dynamic variation of the aggregate force. It is calculated as the ratio of the standard deviation of the aggregate tyre forces to their mean force $[9,13]$. The aggregate tyre force history of a vehicle is the sum of dynamic forces generated by all the tyres, applied to each location along the section, in both wheel paths, raised to the power of (1) since permanent deformation (rutting) is the dominant failure mode for these pavements. The Equivalent Standard Axles (ESA) of dynamic loads for the whole vehicle, averaged over the whole section, were also determined using the fourth power law. The percent changes in ESA of dynamic loads from the ESA of static load, under legal mass limits, were also determined for each section. The latter was regressed against corresponding HATI values but found to produce a weaker relationship than DAFC. Hence, this measure was excluded from further analysis.

The calculated DAFC values associated with each axle group and the whole vehicle for the test sections are plotted in Fig. 4 against their corresponding HATI values. The figure indicates that they generally increase with HATI. The DAFC values for the test sections are within the ranges reported in Cebon [9] for well-damped air suspensions. Lower values of this measure mean better performance or lower damaging potential. For all the sections, DAFC values for the whole vehicle are lower than for the individual axle groups. This could be related to the averaging effect and load

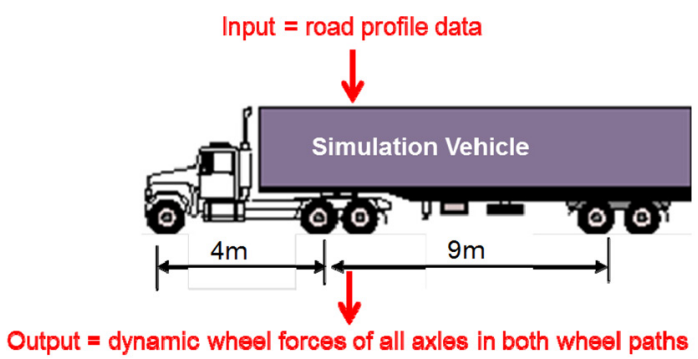

Figure 3: Schematic of the heavy articulated vehicle used in DWL simulation. 


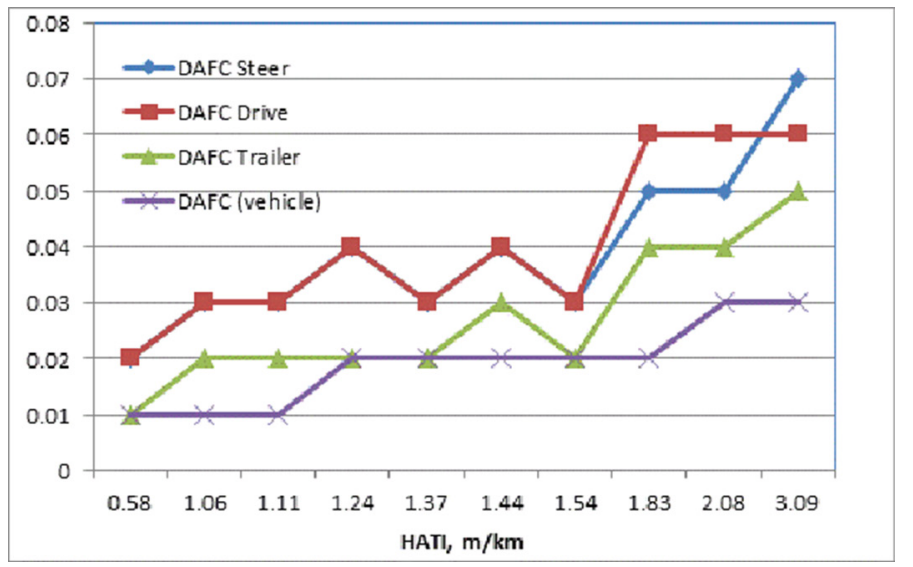

Figure 4: DAFC values for each axle group and the whole vehicle.

Table 1: Correlation coefficients between HATI and IRI and wavelet energies in different wavebands with DAFC for the 10 test sections.

\begin{tabular}{|c|c|c|c|c|c|c|c|c|}
\hline & \multirow[b]{2}{*}{ HATI } & \multirow[b]{2}{*}{ IRI } & \multicolumn{6}{|c|}{ Wavelet energies in wavebands D3-D8 } \\
\hline & & & $\mathrm{Ed} 3$ & Ed4 & Ed5 & Ed6 & $\mathrm{Ed} 7$ & Ed8 \\
\hline DAFC & 0.95 & 0.81 & -0.53 & -0.25 & 0.34 & 0.84 & 0.85 & 0.87 \\
\hline HATI & 1 & 0.92 & -0.49 & -0.14 & 0.36 & 0.88 & 0.81 & 0.77 \\
\hline IRI & 0.92 & 1 & -0.54 & -0.12 & 0.35 & 0.73 & 0.54 & 0.57 \\
\hline
\end{tabular}

transfer by pitching from one axle to another [14]. It ranks the drive axle group as more damaging than the trailer axle group at all HATI values, but similar to or more damaging than the steer axle, at high HATI values.

Table 1 presents the correlation coefficients between DAFC values of the test sections and their corresponding IRI and HATI values. The correlation with HATI is higher than with IRI and this was further confirmed when the sample size was increased. The latter is done by segmenting the profile data of the 10 test sections and their DWL signals into $100 \mathrm{~m}$ subsections and their corresponding HATI, IRI and DAFC values are determined. Figure 5 shows the best regression models for DAFC as a function of HATI and IRI, respectively, for the 37 subsections. It can be noticed that HATI is a much better predictor of DAFC than the IRI. This could be explained by the higher sensitivity of its filter (quarter truck model) to the long wavelengths of roughness spectrum than the IRI filter. This implies that it could be successfully used for identifying sections subject to high DWL, but further testing is required first to confirm.

\subsection{Plots of surface profile DWT and DWL signals}

DWT is performed using a Matlab code to decompose the longitudinal profile data of the 10 test sections into different wavebands. For the sections tested herein, the maximum level of decomposition ranges between 10 and 13. The energies (Ed) corresponding to the different wavebands were 


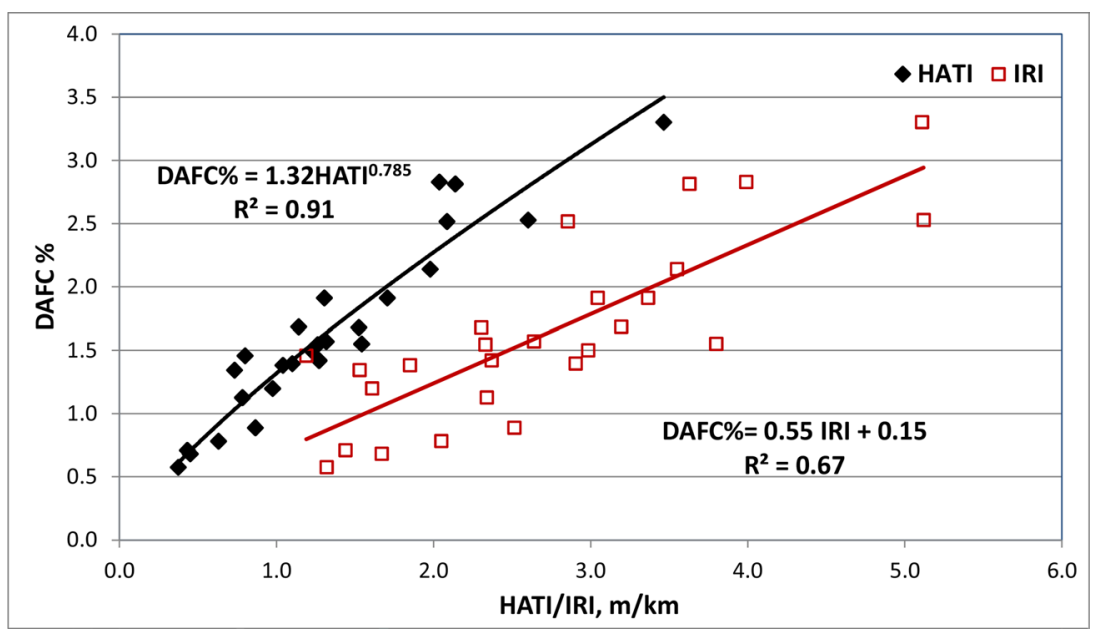

Figure 5: Best regression models for DAFC with HATI and IRI.

correlated with corresponding HATI, IRI and DAFC values. The wavebands that are considered include D3-D8 since they cover wavelengths related to surface roughness $(0.3-30 \mathrm{~m})$ that affect ride perception, i.e. to which HATI and IRI filters are most sensitive. The correlation results are presented in Table 1, which shows that the wavebands with the highest coefficients are D6, D7 and D8.

The signals of these wavebands, in both wheel paths for two of the test sections (300 and $200 \mathrm{~m}$ long), are used to identify and locate the sources of DWL excitations along their lengths. The sections were selected to have, medium and high HATI values. The difference between the signals of the two wheel paths in any waveband represents a roll excitation input and their average represents a bounce excitation input. The signals of roll and bounce excitation inputs in wavebands D6, D7 and D8 for section $1($ HATI $=1.44 \mathrm{~m} / \mathrm{km})$ and section $2($ HATI $=3.09 \mathrm{~m} / \mathrm{km})$ are presented in Figs 6 and 7, respectively, together with corresponding DWL signals. The latter is presented in terms of the ratio of total vehicle DWL (both wheel paths) to static load $(\sim 39 \mathrm{KN})$ at each point along the length of the section.

Careful examination of the two figures revealed the following:

- Generally, the concentration of profile signals energies and peaks in the wavebands 3-26 m (wavebands D6-D8) match with DWL signals variations, keeping in mind that there is a shift between the source of DWL excitation and its impact. The DWL signal combines the contributions of all axle groups, with variable shifts in impacts and contributions along the section. A summary of general observations is provided below.

- Section 1: The highest DWL occurs at the start of the section, 0-35 $\mathrm{m}$ due to bounce and roll inputs (higher from the first) that are evident in the three wavebands. The next peak is between 230 and $240 \mathrm{~m}$ and the excitation inputs are evident in all three wavebands.

- Section 2: The highest DWL occurs between 10 and $30 \mathrm{~m}$, and the signals in the three wavebands, more evident in D6 and D7, clearly indicate a concentration of input from bounce and roll. The latter also corresponds to the concentration of DWL between 90 and $140 \mathrm{~m}$.

The findings from the analyses presented herein indicate that, for the sections tested herein, the highest contribution to the DWL generated at the truck tyre-pavement interface is from the 

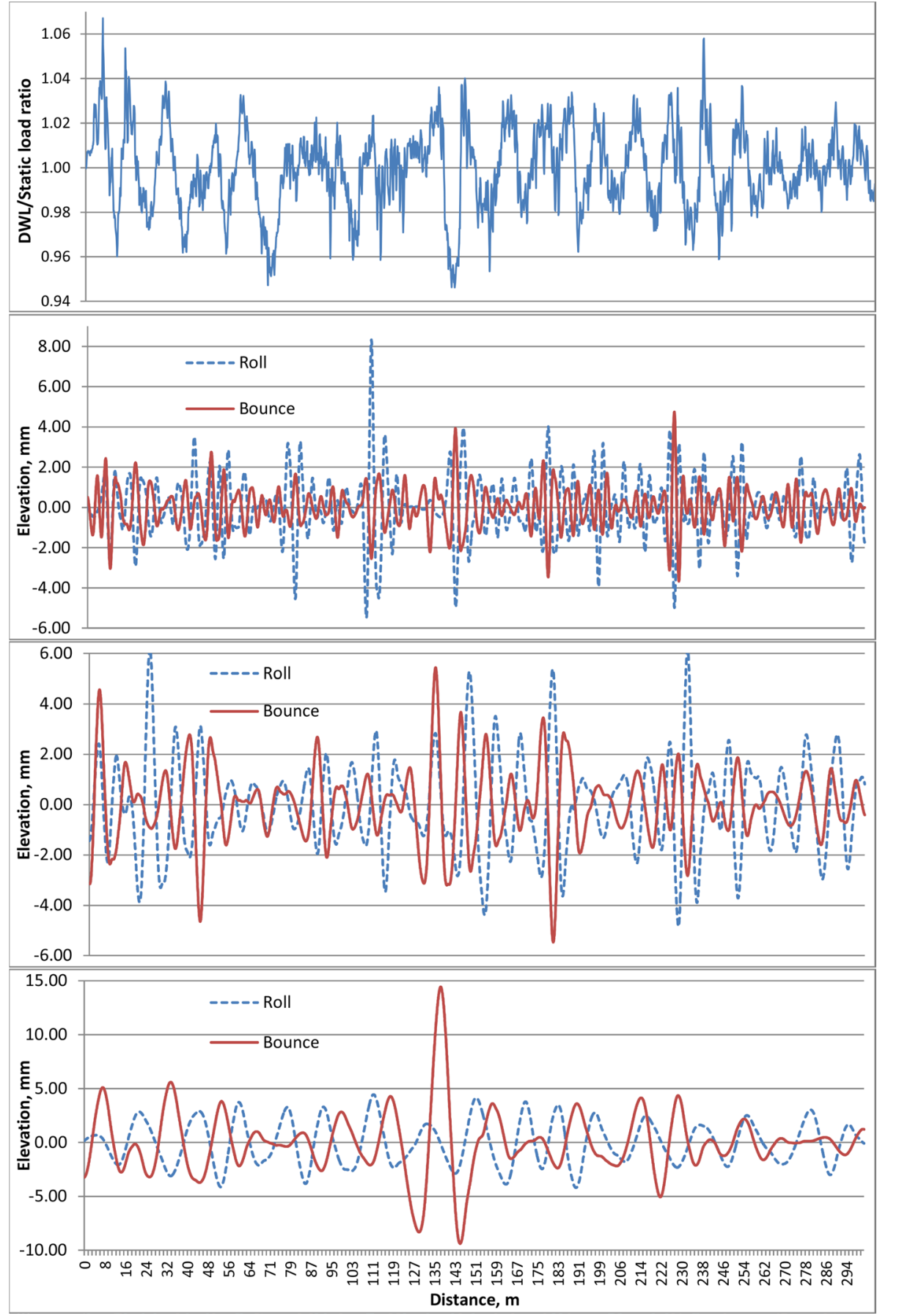

Figure 6: DWL to static load ratio and roll and bounce excitation inputs in D6, D7 and D8, Respectively, for section no. 1. 


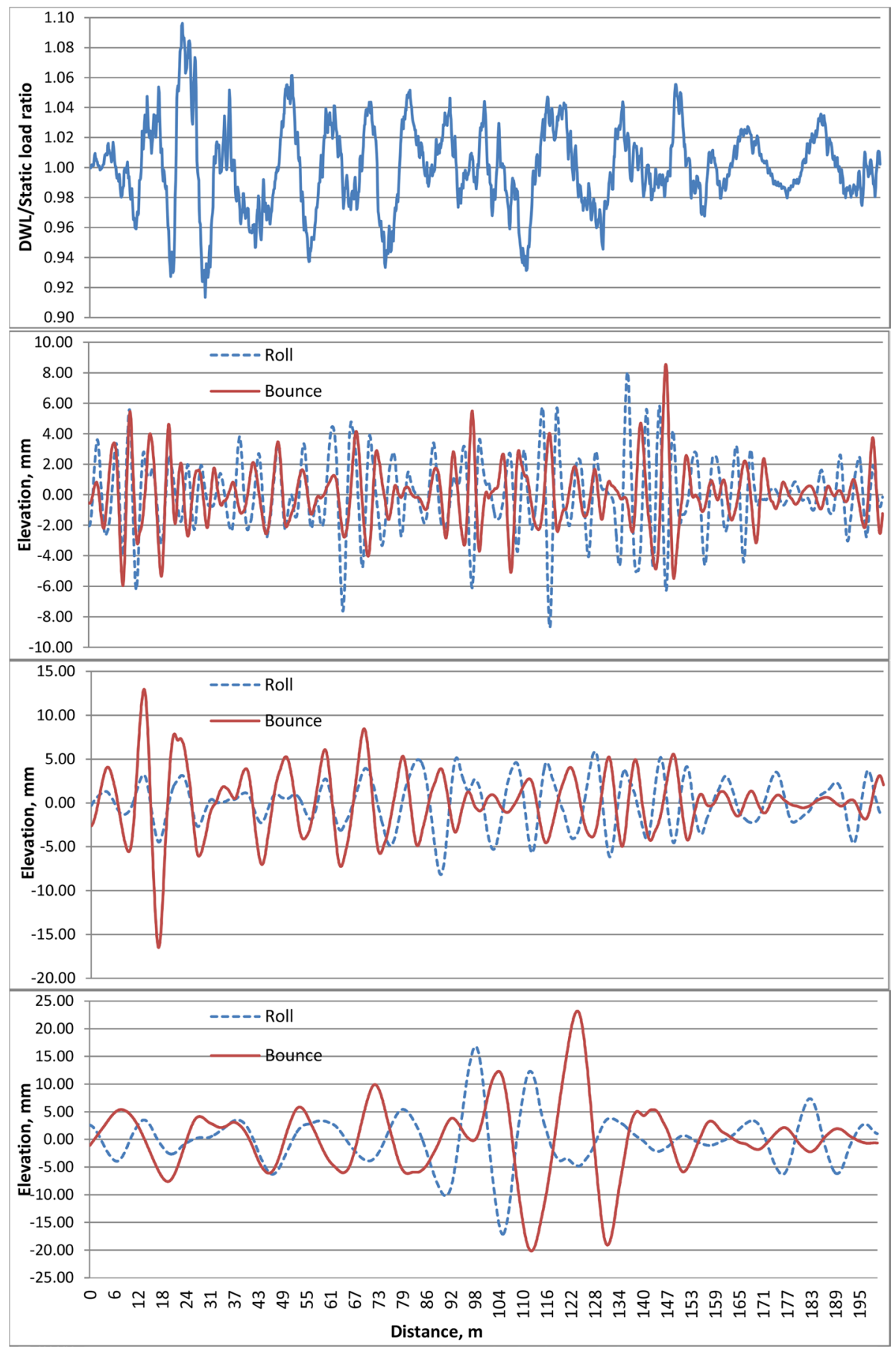

Figure 7: DWL to static load ratio and roll and bounce excitation inputs in D6, D7 and D8, respectively, for section no. 2 . 
low-frequency vibration modes, namely body bounce, pitch and roll. These findings are confirmed with wavelet analysis that has proven to be useful in identifying and locating the sources of DWL excitation inputs along the pavement sections tested herein. The approach proposed herein, when confirmed with further testing, would be useful to road agencies for managing their pavement assets at both network and project levels.

\section{CASE STUDY 2: ASSESSING EFFECTIVENESS OF REHABILITATION TREATMENT}

Asset managers often rely on pavement condition data such as IRI, HATI, rutting, etc. before and after rehabilitation to assess the effectiveness of different treatments. However, this approach is not always sufficient for a detailed assessment of quality of works applied, as demonstrated by this limited case study. A highway section, 900-m long was triggered for rehabilitation to improve ride quality for heavy freight operators as HATI exceeded the threshold of $1.7 \mathrm{~m} / \mathrm{km}$ [1]. The treatment applied is granular overlay. Application of the granular overlay involves removing the existing pavement material to a depth $>100 \mathrm{~mm}$, then a new granular material (100 $\mathrm{mm}$ thick) is laid followed by the application of a new wearing course in the form of a sprayed bitumen seal. The section has been treated in 2007 so its profile data from 2006 and 2008 have to be extracted to conduct the assessment using DWT analysis.

The original profile signals from both years, from which profile data for this section are extracted, are adjusted first to have their starts and ends match. With this approach, it is warranted that the same $900 \mathrm{~m}$ section is being compared in both years. This is done using Proval [15] to view the profiles and determine the shifts required. The adjustments are then applied by changing the sampling interval using an in-house Excel-based tool [16]. Average HATI values of the test section before and after treatment are 1.72 and $0.44 \mathrm{~m} / \mathrm{km}$, respectively, and average IRI values were 3.78 and $1.42 \mathrm{~m} / \mathrm{km}$, respectively. The section is then segmented into 10 subsections $(90 \mathrm{~m}$ each) for use in performing DWT analysis. The reason for segmentation is that it makes it easier to observe the changes at different locations in the different wavebands.

DWT signals in D3-D7 for the outer wheel paths of two of these subsections, before and after treatment, are plotted in Figs 8 and 9, together with corresponding energy values. HATI values for subsection A (Fig. 8) before and after treatment are 1.14 and 0.40, respectively, and 2.54 and $1.37 \mathrm{~m} / \mathrm{km}$ for IRI, respectively. For subsection B (Fig. 9), they are 1.94 and 0.44 for HATI and for IRI, 3.54 and $1.55 \mathrm{~m} / \mathrm{km}$, respectively. The reduction in profile elevations after treatment is evident in both figures; however, there are locations (circled) where the elevation variations posttreatment are higher than before. The differences between energy values of each waveband in 2006 (Ed06) and 2008 (Ed08) also indicate the extent of reduction in elevation variations in the different wavebands due to the application of treatment. They are lower in the wavebands where the plots indicate higher elevation variations posttreatment (circled locations).

Figure 10 presents the bounce and roll inputs for sub-section B in waveband D6. It clearly shows that the application of treatment has not been successful in eliminating the source of bounce excitation around the 40-m location. However, it has been successful in fixing the elevation variations between the two wheel paths that contribute to the excitation of body roll.

\section{CONCLUSIONS}

The application of wavelet analysis in road pavement management at project level is described through two case studies. The first involves using its outputs (energies and signals) in identifying and locating excitation sources of DWL along a road section. The second case study involves using these outputs in assessing the effectiveness of rehabilitation treatment in reducing surface roughness in the 


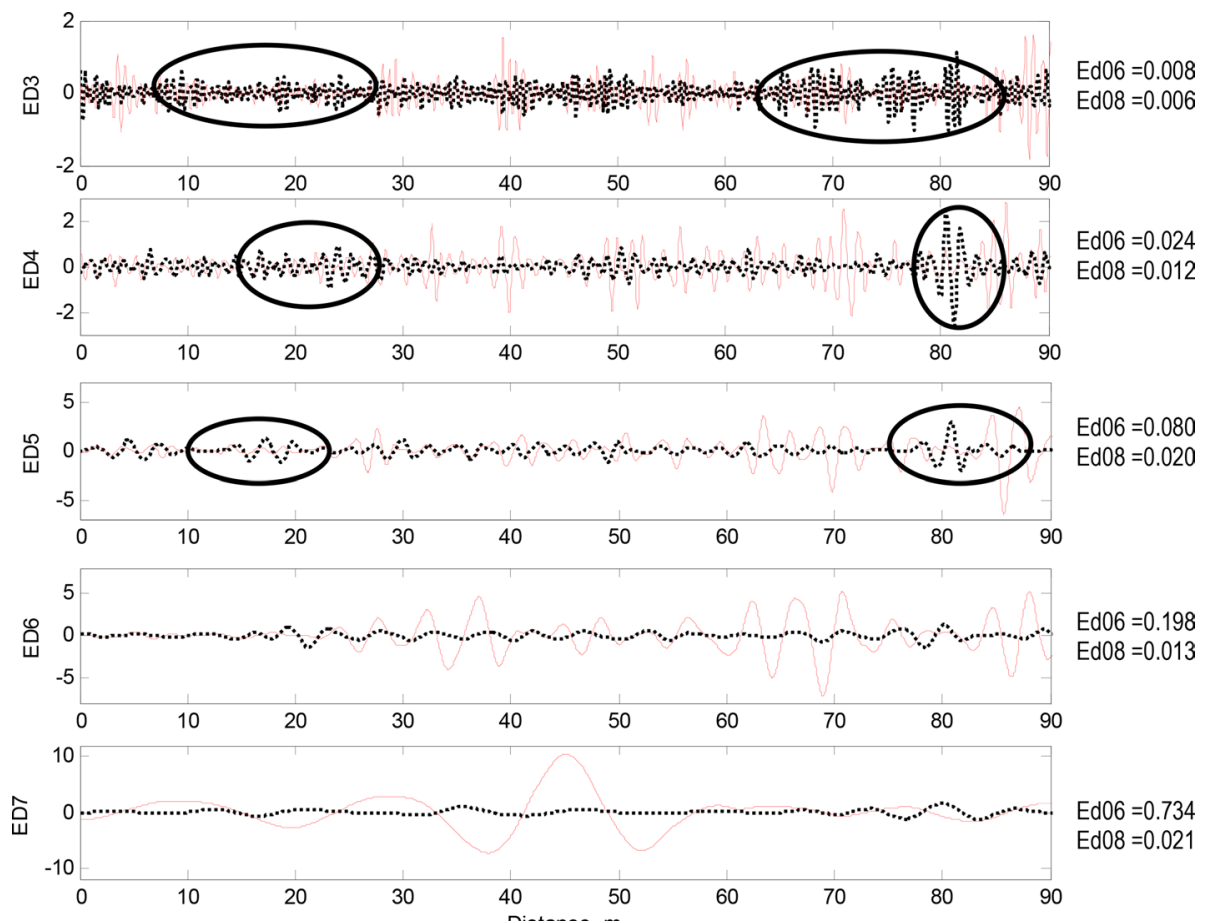

Figure 8: DWT signals in D3-D7 of outer wheel path for subsection A in 2006 (solid lines) and 2008 (dotted lines) and corresponding energies.

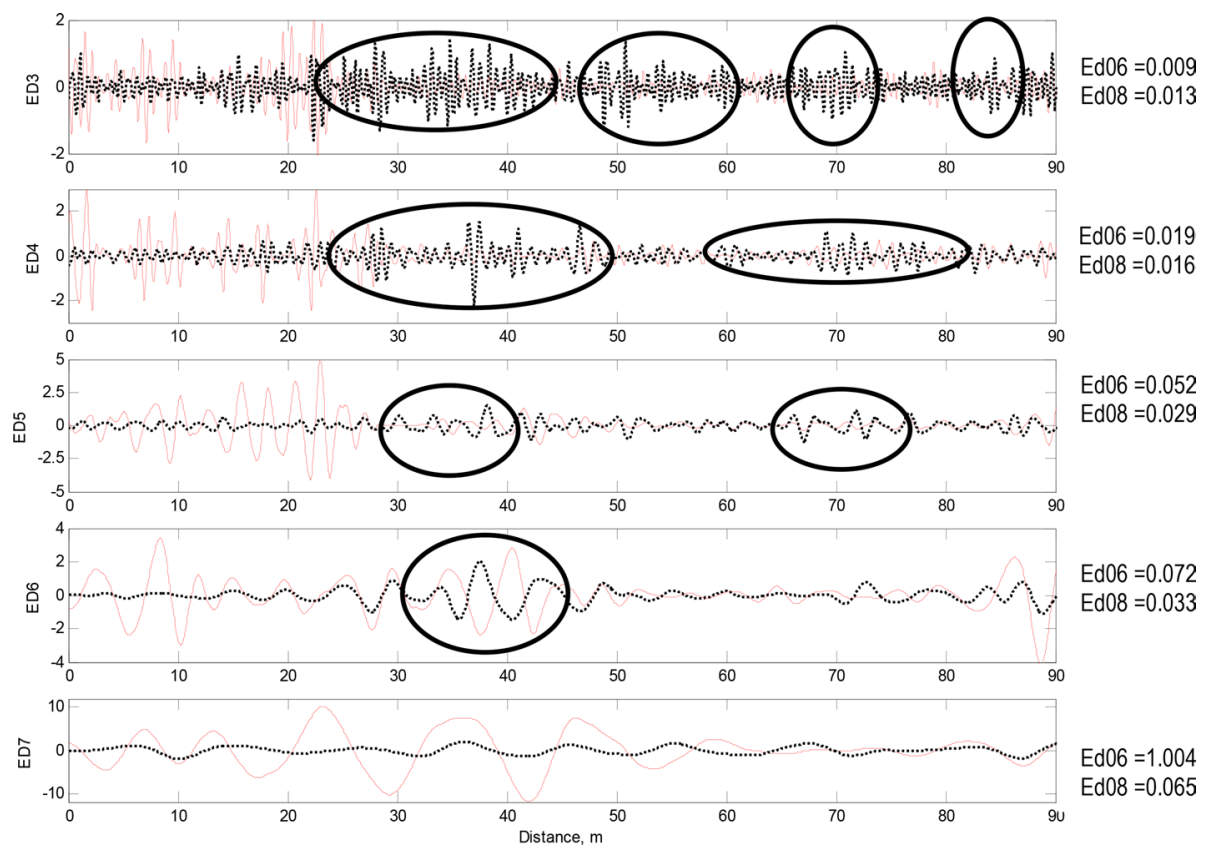

Figure 9: DWT signals in D3-D7 of outer wheel path for subsection B in 2006 (solid lines) and 2008 (dotted lines) and corresponding energies. 

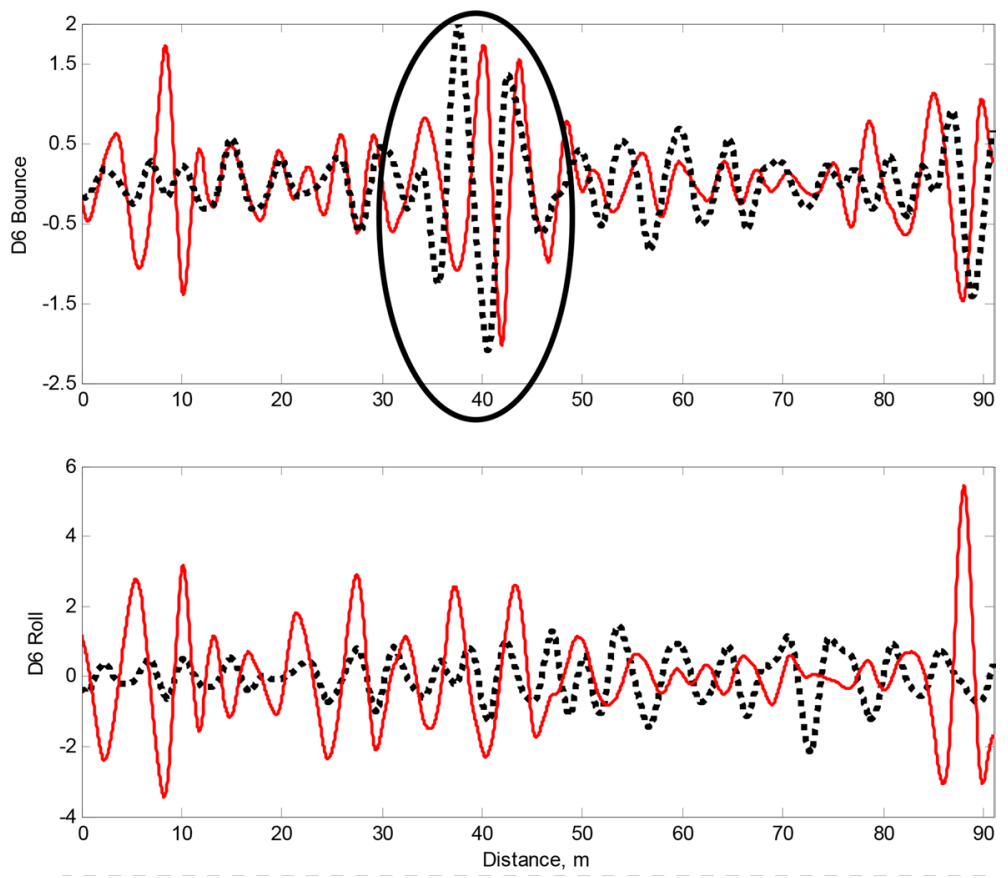

Figure 10: Bounce and roll inputs for D6 of subsection B in 2006 (solid) and 2008 (dotted).

different wavebands along the length of a road section. The outcomes of this research study indicate that the proposed applications are effective in addressing their intended purposes.

Study findings also indicate that using HATI to highlight sections subject to high DWL at network level is promising. However, further testing is required to confirm its suitability at different speeds and operating environments. It should include a larger sample size of sections covering a wider range of operating and environmental conditions.

Additionally, simulation models of DWL should be representative of the current trends of heavy vehicle fleet, i.e. longer and heavier trucks such as B-Doubles. However, it is important to note that outputs of simulation models using single truck models will always be indicative only. The reason is that trucking fleet consists of a variety of trucks with different wheelbase lengths and load limits. This results in many points at different locations along the road being subjected to repeated high DWL due to spatial repeatability. These points are not depicted with single truck models.

In terms of practical application, the use of DWT for assessing treatment effectiveness of a project is feasible. Surface profile data of the treated sections are normally collected to determine roughness values posttreatment so all that is needed is a computer program for running wavelet analysis. As for identifying sections subject to high DWL and locate sources of excitation, HATI can be used as an indicator of such sections and confirmed with wavelet analysis. Such sections can be further assessed using DWT when programmed for rehabilitation to locate sources of DWL excitation. Profile data of the road network is collected regularly for performance monitoring, so data of such sections can be extracted and analysed. This approach helps in determining appropriate remedial measures to ensure all sources of excitation are eliminated. 


\section{REFERENCES}

[1] Hassan, R. \& McManus, K., Sustainable mobility for heavy freight vehicles. International Journal of Sustainable Development and Planning, 5(3), pp. 253-268, 2010. doi: http://dx.doi. org/10.2495/sdp-v5-n3-253-268

[2] Doré, G., Flamand, M. \& Pascale, P., Analysis of the wavelength content of the longitudinal profiles for C-LTPP test sections. Canadian Journal of Civil Engineering, 29(1), pp. 50-57, 2002. doi: http://dx.doi.org/10.1139/101-075

[3] Brown, D., Liu, W. \& Henning, T.F.P., Identifying pavement deterioration by enhancing the definition of road roughness, Research Report 430. NZTA, NZ Transport Agency Wellington, 2010.

[4] Delanne, Y. \& Pereira, P.A.A., Advantages and limits of different road roughness profile signal-processing procedures applied in Europe. Transportation Research Record, 1764, pp. 254-259, 2001. doi: http://dx.doi.org/10.3141/1764-26

[5] Liu,W. \& Fwa, T.F., Application of wavelet transform analysis for pavement roughness studies. Proceedings of the Eighth International Conference on Applications of Advanced Technologies in Transportation Engineering, Beijing, pp. 455-459, 2004. doi: http://dx.doi.org/ $\underline{10.1061 / 40730(144) 85}$

[6] Papagiannakis, A.T., Zelelew, H.M. \& Muhunthan, B., A wavelet interpretation of vehiclepavement interaction. International Journal of Pavement Engineering, 8(3), pp. 245-252, 2007. doi: http://dx.doi.org/10.1080/10298430701309378

[7] Wasilewski, F., Wavelet Broswer by Pywavelets, available at http://wavelets.pybytes.com/, 2011 (accessed July 2013).

[8] Sweatman, P.F., A study of the dynamic wheel forces in axle group suspensions of heavy vehicles, ARRB Special Report No. 27, Melbourne, Australia, 1983.

[9] Cebon, D., Handbook of Vehicle Road Interaction, Swets \& Zeitlinger Publishers: London, 1999.

[10] OECD, Dynamic Interaction of Vehicle and Infrastructure Experiment (OECD IR6 Project: Dynamic Interaction of Heavy Vehicles with Roads and Bridges, DIVINE Project). Proceedings of the Asia-Pacific Concluding Conference, Melbourne, Australia, 1997.

[11] De Pont, J., Road Profile Characterisation, Transit New Zealand Research Report No. 29, 1994.

[12] Papagiannakis, A.T. \& Gujarathi, M.S., Roughness Model Describing Heavy Vehicle-Pavement Interaction, Transportation Research Record 1501, pp. 50-59, 1995.

[13] Cole, D.J. \& Cebon, D., Influence of tractor-trailer interaction on assessment of road damaging performance. Proceedings of the Institution of Mechanical Engineers, Part D, Journal of Automobile Engineering, 212, p. 1, 1998. doi: http://dx.doi.org/10.1243/0954407981525759

[14] Bernard, J. \& Dolcemascolo, V., Dynamic interaction between instrumented vehicles and pavements. Fifth International Symposium on Heavy Vehicle Weights and Dimensions, Australia, 1998.

[15] Proval, http://www.roadprofile.com/

[16] Evans, R. \& Arulrajah A., A new method for aligning and synchronising road profile data for better road roughness growth analysis. 8th International Conference on Managing Pavement Assets, CD-ROM, Paper ICMPA011, 15-19 November, Santiago, Chile, 2011. 